\title{
Modeling of the ORNL PCA Benchmark Using SCALE6.0 Hybrid Deterministic-Stochastic Methodology
}

\author{
Mario Matijević, Dubravko Pevec, and Krešimir Trontl \\ University of Zagreb, Faculty of Electrical Engineering and Computing, Department of Applied Physics, Unska 3, 10000 Zagreb, Croatia \\ Correspondence should be addressed to Krešimir Trontl; kresimir.trontl@fer.hr
}

Received 30 May 2013; Accepted 29 August 2013

Academic Editor: Valerio Giusti

Copyright ( 2013 Mario Matijević et al. This is an open access article distributed under the Creative Commons Attribution License, which permits unrestricted use, distribution, and reproduction in any medium, provided the original work is properly cited.

\begin{abstract}
Revised guidelines with the support of computational benchmarks are needed for the regulation of the allowed neutron irradiation to reactor structures during power plant lifetime. Currently, US NRC Regulatory Guide 1.190 is the effective guideline for reactor dosimetry calculations. A well known international shielding database SINBAD contains large selection of models for benchmarking neutron transport methods. In this paper a PCA benchmark has been chosen from SINBAD for qualification of our methodology for pressure vessel neutron fluence calculations, as required by the Regulatory Guide 1.190. The SCALE6.0 code package, developed at Oak Ridge National Laboratory, was used for modeling of the PCA benchmark. The CSAS6 criticality sequence of the SCALE6.0 code package, which includes KENO-VI Monte Carlo code, as well as MAVRIC/Monaco hybrid shielding sequence, was utilized for calculation of equivalent fission fluxes. The shielding analysis was performed using multigroup shielding library v7_200n47g derived from general purpose ENDF/B-VII.0 library. As a source of response functions for reaction rate calculations with MAVRIC we used international reactor dosimetry libraries (IRDF-2002 and IRDF-90.v2) and appropriate crosssections from transport library v7_200n47g. The comparison of calculational results and benchmark data showed a good agreement of the calculated and measured equivalent fission fluxes.
\end{abstract}

\section{Introduction}

Calculational methods for determining the neutron fluence are necessary to estimate the fracture toughness of the reactor pressure vessel (RPV) materials, which is one of the key requirements in determining operational limits and lifetime of nuclear power plants. This area of research is of a particular importance in the era of plant lifetime extension demands and possible financial savings which could be achieved by approving the extensions. Any developed or analyzed calculation methodology requires comprehensive verification and validation against evaluated reference data. A large database of benchmarks aimed at validation of computer codes and nuclear data used for radiation transport and shielding problems is "Shielding Integral Benchmark Archive and Database (SINBAD)" [1]. One of the most widely used SINBAD benchmarks for qualification of radiation transport methods and evaluation of appropriate nuclear data used for transport as well as for dosimetry calculations in Light Water Reactors (LWR) is the "Pool Critical Assembly Pressure Vessel Facility Benchmark" (PCA benchmark) [2].
The purpose of the benchmark was to validate the capabilities of the calculational methodology to predict the reaction rates in the region outside the reactor core when the neutron source, material compositions, and geometry are well defined. Over the years a number of PCA benchmark studies have been conducted. Their classification is possible based on the type of neutron transport calculation (discrete ordinates method or Monte Carlo method), as well as nuclear data libraries used either for neutron transport or dosimetry calculations.

Benchmark original calculational results obtained by discrete ordinates synthesis transport method through DORT and DOTSYN codes and BUGLE-93, SAILOR-95, and BUGLE-96 nuclear data libraries for transport calculation and CROSS-95 library for dosimetry calculations were improved by Fero et al. [3] using full 3D discrete ordinates transport method and BUGLE-96 library. Improvement of calculated to measured ratio $(\mathrm{C} / \mathrm{M})$ was observed and attributed to full 3D approach. Lee [4] used 3D TRIPOLI4.3 Monte Carlo code with continuous energy ENDF/B-VI.4 
and JEF 2.2 nuclear data libraries for neutron transport and IRDF-90, IRDF-90.v2, RRDF-98, and JENDL/D-99, as well as ENDF/B-VI.4 and JEF 2.2 nuclear data libraries for dosimetry calculations. The author finds the IRDF-90 file generally good for RPV dosimetry calculations but recommends ${ }^{103} \mathrm{Rh}(\mathrm{n}$, $\left.\mathrm{n}^{\prime}\right){ }^{103 m} \mathrm{Rh}$ and ${ }^{237} \mathrm{~Np}(\mathrm{n}, \mathrm{f}){ }^{137} \mathrm{Cs}$ dosimetry cross-sections from RRDF-98 and JENDL/D-99 libraries.

An initial analysis of the applicability of SCALE6.0 [5] hybrid deterministic-stochastic methodology for the PCA benchmark analysis was conducted by Vragolov et al. [6]. Only a preliminary averaged equivalent fission fluxes were calculated, and the results showed SCALE6.0 possibilities but also raised issues that required further analysis. The main concern was the choice of nuclear data library used for dosimetry calculation.

Recently Risner et al. [7] used SCALE6.0 code package for validation of VITAMIN-B7 and BUGLE-B7 nuclear data libraries on a number of benchmarks, including PCA benchmark. The overall results showed applicability of both libraries with SCALE6.0 sequences for PCA benchmark analysis, with exception of ${ }^{103} \mathrm{Rh}\left(\mathrm{n}, \mathrm{n}^{\prime}\right){ }^{103 m} \mathrm{Rh}$ and ${ }^{115} \mathrm{In}(\mathrm{n}$, $\left.\mathrm{n}^{\prime}\right)^{115 m}$ In dosimetry cross-sections. The authors advice usage of IRDF-2002 library for named dosimeter reactions.

We believe that additional investigation of SCALE6.0 capabilities could be useful, primarily in the context of direct application of SCALE6.0 built-in fine-group data libraries for neutron transport, as well as for dosimetry calculations. Such an approach would speed up analysis, and it would avoid incorporation of external cross-section libraries into SCALE6.0 sequences which is not a straight-forward procedure, especially for inexperienced users. Therefore, we conducted PCA benchmark analysis using SCALE6.0 code package with built-in fine-group nuclear data libraries, namely v7_238 [5] for criticality calculation using CSAS6 and v7_200n47g [5] for shielding calculations using MAVRIC sequence. Dosimetry cross-section data were extracted from nuclear data library used for transport calculation (v7_200n47g). Since we faced similar problems as Risner et al. with data for ${ }^{103} \mathrm{Rh}\left(\mathrm{n}, \mathrm{n}^{\prime}\right)^{103 m} \mathrm{Rh}$ and ${ }^{115} \operatorname{In}\left(\mathrm{n}, \mathrm{n}^{\prime}\right)^{115 m} \mathrm{In}$, we used IRDF-2002 [8] and IRDF-90.v2 [9] dosimetry libraries for all dosimeters in order to examine the behavior of every PCA benchmark dosimeter reaction with different library.

The PCA benchmark is described in Section 2. The description of the SCALE6.0 code package used for modeling of the PCA benchmark and calculational methodology are given in Section 3. The analysis of the PCA benchmark, including results of calculation, and comparison of calculated values with benchmark data is presented in Section 4, while the conclusions are given in Section 5. References are given at the end of the paper.

\section{PCA Benchmark Facility Description}

The main cause for limiting the PWR power plant lifetime is the fast neutron fluence induced embrittlement of the reactor pressure vessel (RPV). With the advances of computer computational power the reactor dosimetry calculations give better insight in radiation damage of RPV when exposed to intense neutron flux. Correlations can be made between neutron flux and irradiation of in-core and ex-core detectors, typically via displacements per atom (DPA). This is important for advanced nuclear material selection, probing and scoping material behavior in intense radiation environments, for example, gas accumulations in reactor baffle plates by ${ }^{58} \mathrm{Ni}(\mathrm{n}$, $\gamma)^{59} \mathrm{Ni}(\mathrm{n}, \alpha)$ reaction sequence. The current guideline for RPV dosimetry calculations is the US NRC Regulatory Guide 1.190 [10], which states that calculational methods used to estimate RPV fast fluence should use the latest version of the Evaluated Nuclear Data File (ENDF/B) in fast energy range (0.1-15) MeV. In accordance with this guideline we present calculational results for ORNL PCA Benchmark.

The scope of PCA benchmark is to validate the capabilities of the calculational methodologies to predict the reaction rates in the region outside the core when the neutron source, material compositions, and relatively simple geometry configuration are well defined and given. The PCA benchmark provides calculated and measured reaction rates $(\mathrm{C} / \mathrm{M}$ ratio) inside the simulated pressure vessel, as well as in the water gap in front of the pressure vessel. This allows an assessment of the accuracy with which the calculations predict the neutron flux attenuation inside the pressure vessel.

The PCA benchmark facility consists of the reactor core and the components that mock up the reactor-to-cavity region in light water reactors. These components are the thermal shield (TS), the reactor pressure vessel simulator (RPVS), and the void box (VB), which simulates the reactor cavity. An overall view of the PCA benchmark facility is shown in Figure 1. An aluminum plate, referred to in Figure 1 as the reactor window simulator, was added to the facility for operational reasons. The thicknesses of the water gaps between the aluminum window and thermal shield and between the thermal shield and pressure vessel are approximately $12 \mathrm{~cm}$ and $13 \mathrm{~cm}$, respectively. Such configuration is known as 12/13 configuration. The materials used for the components outside the core were aluminum for the reactor window simulator, stainless steel for the thermal shield, and carbon steel for the pressure vessel. The facility is located in a large pool of water, which serves as reactor core coolant and moderator and provides shielding. The PCA benchmark facility core is a light water moderated, enriched uranium fueled critical assembly. It consists of 25 material test reactor (MTR) plate type elements. The standard MTR fuel element and control element are depicted in Figure 2. The eight vertical experimental access tubes in which the measurements were done were filled with appropriate material (steel in the pressure vessel locations and Plexiglas in the in-water locations) in order to minimize the perturbations of the neutron field.

Measured quantities, used in PCA benchmark, are given in terms of the equivalent ${ }^{235} \mathrm{U}$ fission fluxes which were calculated by dividing the reaction rates with the cross-sections averaged over the ${ }^{235} \mathrm{U}$ fission spectrum [2]. All measured quantities provided for comparison with calculated values are given per unit PCA benchmark facility core neutron source. Therefore, the calculated results need to be normalized to the source strength of one fission neutron per second being 


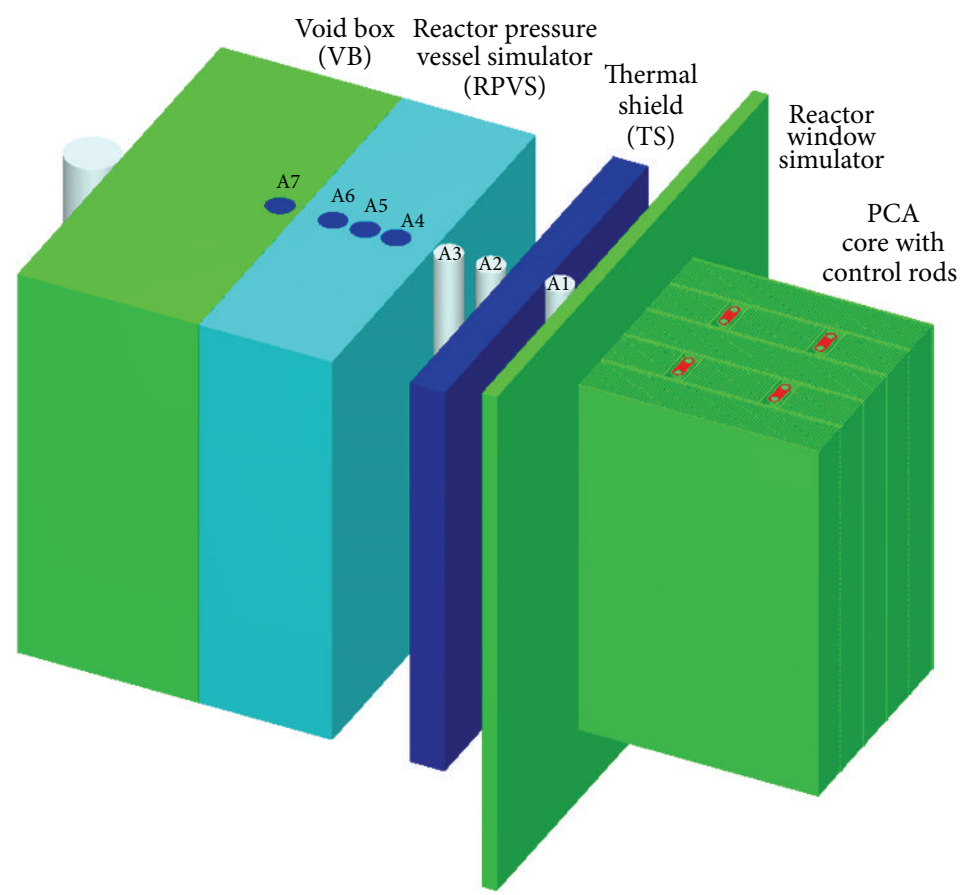

FIGURE 1: SCALE6.0 model of PCA benchmark facility (water removed).
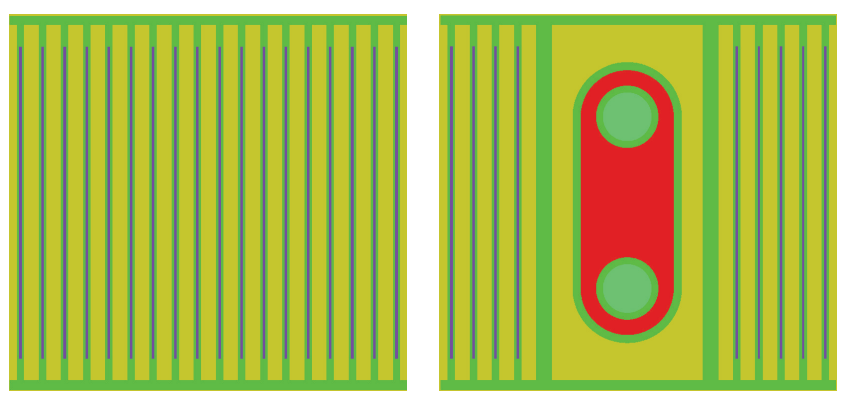

FIGURE 2: PCA standard MTR fuel and control element (water included).

born in the whole PCA core. The ratios of the calculated-tomeasured $(\mathrm{C} / \mathrm{M})$ equivalent fission fluxes for DORT libraries BUGLE-93, SAILOR-95, and BUGLE-96 are given in PCA benchmark reference [2]. Measurements were performed at core midplane $(z=0)$ at several locations, labeled in Figure 1 as A1 to A7. To complete the PCA benchmark analysis the analyst must determine the calculated-to-measured (C/M) ratios of the equivalent ${ }^{235} \mathrm{U}$ fission fluxes for all the locations and all the dosimeters for which the measured values are provided.

The significance of the PCA Benchmark are experimental data measurements inside thick steel RPV in locations A4 to A6, that is, neutron flux gradient inside the pressure vessel, which provides the means for verification of calculated neutron flux attenuation. This is in contrast to available data from operating reactors, which are typically addressing neutron flux for downcomer region internal to RPV and reactor cavity external to RPV wall [11].

\section{SCALE6.0 Code Package}

The SCALE6.0 [5] code system was developed for the US NRC to satisfy a need for a standardized method of analysis for the evaluation of nuclear facilities and package designs. In its present form, the system has the capability to perform criticality, shielding, radiation source term, spent fuel depletion/decay, reactor physics, and sensitivity analyses using well established functional modules tailored to the SCALE6.0 system.

3.1. The CSAS6 Sequence. The CSAS6 criticality sequence is developed to provide automated, problem-dependent, crosssection processing followed by calculation of the neutron multiplication factor $k_{\text {eff }}$. The cross-section processing code BONAMI is used for the unresolved resonance range (Bondarenko factors) and either NITAWL or WORKER/CENTRM/PMC for the resolved resonance range. KENO-VI, a 3D multigroup Monte Carlo code, is its functional module.

3.2. The MAVRIC Sequence. The MAVRIC shielding sequence uses automated hybrid deterministic-stochastic methodology to generate variance reduction (VR) parameters for Monte Carlo calculations. MAVRIC uses 3D neutron transport code Monaco with integrated $S_{N}$ code Denovo, and it is based on CADIS (Consistent Adjoint Driven Importance Sampling) [12] and FW-CADIS methodology [13]. Denovo is used in forward and adjoint mode to approximate space-energy flux and adjoint function, respectively. These solutions are utilized to calculate space and energy dependent biasing parameters, that is, biased 
source and transport importance map, to be used as a VR in Monaco. CADIS is used to optimize results in localized regions of phase-space, while FW-CADIS tends to obtain global uniform statistical uncertainty by weighting of the adjoint source with expected detector response approximated with forward Denovo solution. CADIS and FW-CADIS are based on the adjoint function [14] (i.e., solution of the adjoint Boltzmann equation) which has long been recognized as the importance function for some objective function of interest. Detector response is found by integrating the product of the detector cross-section $\sigma_{d}(\vec{r}, E)$ and flux over detector volume:

$$
R=\int_{V_{D}} \int_{E} \sigma_{d}(\vec{r}, E) \phi(\vec{r}, E) d V d E .
$$

Alternatively, if we approximate adjoint scalar flux with quick Denovo solution, where the adjoint source is set as $q^{\dagger}(\vec{r}, E)=$ $\sigma_{d}(\vec{r}, E)$, then the detector response is found by integrating over source volume:

$$
R=\int_{V_{S}} \int_{E} q(\vec{r}, E) \phi^{\dagger}(\vec{r}, E) d V d E .
$$

The biased source distribution [15], which minimizes the variance of user-desired response, is given as

$$
\widehat{q}(\vec{r}, E)=\frac{\phi^{\dagger}(\vec{r}, E) q(\vec{r}, E)}{R},
$$

where $\phi^{\dagger}(\vec{r}, E), q(\vec{r}, E)$, and $R$ are the scalar adjoint function, the source emission probability (forward source), and total detector response from (2), respectively. For transport biasing the weight window technique is employed, that is, spaceenergy dependent geometric splitting/roulette. Biased source and weight-window lower bounds are consistent, so the source particles are created with statistical weight within weight windows:

$$
\bar{w}(\vec{r}, E)=\frac{q(\vec{r}, E)}{\widehat{q}(\vec{r}, E)}=\frac{R}{\phi^{\dagger}(\vec{r}, E)} .
$$

Inverse relationship between particle statistical weight and adjoint function must be emphasized. Since PCA Benchmark involves calculation of near and far detector reaction rates, this FW-CADIS methodology is highly desirable choice.

3.3. Cross-Section Libraries. There are several multigroup cross-section libraries distributed within SCALE6.0 for criticality safety analyses and shielding calculations. For criticality safety analyses the v7-238 library [5] was used, while for the shielding calculations v7-200n47g library [5] was used. They are based on ENDF/B-VII.0 data [16]. For shielding part of calculations it was imperative to use fine structure library v7_200n47g, because high-energy threshold for all reactions could not be correctly accounted for if broad shielding v7_27n19g library was used.

\section{Analysis of the PCA Benchmark}

The calculational models of the PCA benchmark within CSAS6 and MAVRIC sequences of SCALE6.0 code package have been determined. The results of the calculations, that is, equivalent fission fluxes, using the established models have been compared with the PCA benchmark data.

4.1. CSAS6 Results. The initial CSAS6/KENO-VI criticality eigenvalue calculation of the PCA benchmark facility was performed using 550 batches and 2000 neutrons per batch with first 50 batches skipped in order for the fission source distribution to converge to eigenmode. Critical control rod positions were validated and obtained effective multiplication factor of the system was $k_{\text {eff }}=(0.99856 \pm 0.00071)$. This result was tested with MCNP5 code [17], Shannon entropy check for source stationarity was successful and similar $k_{\text {eff }}$ was obtained: $k_{\text {eff }}=(0.99911 \pm 0.00051)$. This CSAS6/KENO-VI calculation was then rerun with better statistics (2150 batches/10000 neutrons per batch) for the generation of mesh-based fission source distribution ("CDS =YES" option), to be used as a criticality source in the subsequent MAVRIC calculations. This approach in SCALE6.0 is known as CAAS (Criticality Accident Alarm Systems).

4.2. MAVRIC/Monaco Results. The MAVRIC shielding sequence was run with the mesh-based source from criticality eigenvalue run. FW-CADIS methodology provides consistent weight windows (i.e., importance map) and biased source distributions for transport of the PCA core neutrons to the locations of interest: detectors in experimental access tubes A1-A7.

The equivalent fission fluxes in PCA report were calculated by dividing the reaction rates by the cross-sections averaged over the ${ }^{235} \mathrm{U}$ fission spectrum. That fast neutron fluence calculation was reasonable approach in the time of PCA Benchmark calculation with DORT, but full spectrum calculation nowadays with modern Monte Carlo code is not prohibitive. So our calculations were conducted with full neutron spectrum in accordance with SCALE6.0 multigroup weighting function (spectrum) [2] defined as

(1) Maxwellian spectrum (peak at $300 \mathrm{~K}$ ) from $10^{-5}$ to $0.125 \mathrm{eV}$,

(2) a $1 / E$ spectrum from $0.125 \mathrm{eV}$ to $67.4 \mathrm{keV}$,

(3) a fission spectrum (effective temperature at 1.273 $\mathrm{MeV}$ ) from $67.4 \mathrm{keV}$ to $10 \mathrm{MeV}$,

(4) a $1 / E$ spectrum from 10 to $20 \mathrm{MeV}$.

Equivalent fission fluxes thus are defined as

$$
\phi_{\mathrm{eq}}=\frac{\int_{E} \sigma_{i}(E) \phi(E) d E}{\int_{E} \sigma_{i}(E) \varphi(E) d E / \int_{E} \varphi(E) d E}=\frac{\text { reaction rates }}{\overline{\sigma_{i}}},
$$

where $\sigma_{i}(E), \phi(E)$ and $\varphi(E)$, are the dosimetry cross-sections for the reactions being considered, the Monte Carlo flux at the dosimetry location, and weighting spectrum function, respectively. The spectrum averaged cross-sections $\left(\overline{\sigma_{i}}\right)$ were taken from Table 1.6 from PCA report [2] for consistency, since they are in very good agreement with calculated values from our libraries. 
For seven detector locations (A1-A7) we used two dosimetry libraries as a source of a response functions for reaction rate calculations. Reaction rate calculations were done with IRDF-2002 and IRDF-90.v2 (older version) libraries, for the total of six PCA benchmark reactions: ${ }^{237} \mathrm{~Np}(\mathrm{n}, \mathrm{f}){ }^{137} \mathrm{Cs},{ }^{238} \mathrm{U}(\mathrm{n}, \mathrm{f}){ }^{137} \mathrm{Cs},{ }^{103} \mathrm{Rh}\left(\mathrm{n}, \mathrm{n}^{\prime}\right){ }^{103 m} \mathrm{Rh},{ }^{115} \mathrm{In}(\mathrm{n}$, $\left.\mathrm{n}^{\prime}\right){ }^{115 m} \mathrm{In},{ }^{58} \mathrm{Ni}(\mathrm{n}, \mathrm{p})^{58} \mathrm{Co}$, and ${ }^{27} \mathrm{Al}(\mathrm{n}, \alpha)^{24} \mathrm{Na}$. These reactions were defined as a response functions for FW-CADIS methodology.

The threshold energies for the ${ }^{27} \mathrm{Al}(\mathrm{n}, \alpha)^{24} \mathrm{Na},{ }^{58} \mathrm{Ni}(\mathrm{n}$, p $)^{58} \mathrm{Co},{ }^{238} \mathrm{U}(\mathrm{n}, \mathrm{f}){ }^{137} \mathrm{Cs},{ }^{237} \mathrm{~Np}(\mathrm{n}, \mathrm{f}){ }^{137} \mathrm{Cs},{ }^{115} \mathrm{In}\left(\mathrm{n}, \mathrm{n}^{\prime}\right){ }^{115 m} \mathrm{In}$, and ${ }^{103} \mathrm{Rh}\left(\mathrm{n}, \mathrm{n}^{\prime}\right){ }^{103 m} \mathrm{Rh}$ reactions are 5.0, 2.05, 1.45, 0.69, 0.3 , and $0.1 \mathrm{MeV}$, respectively. This is in fast neutron range, but still, calculations were performed for full neutron spectrum $\left(10^{-5} \mathrm{eV}\right.$ to $\left.20 \mathrm{MeV}\right)$ with fine-group shielding library v7_200n $47 \mathrm{~g}$.

Also, we investigated SCALE6.0 capabilities in generation/mixing of multigroup cross-sections from v7 200n $47 \mathrm{~g}$ library for reactions ${ }^{237} \mathrm{~Np}(\mathrm{n}, \mathrm{f}){ }^{137} \mathrm{Cs},{ }^{238} \mathrm{U}(\mathrm{n}, \mathrm{f}){ }^{137} \mathrm{Cs},{ }^{58} \mathrm{Ni}(\mathrm{n}$, $\mathrm{p})^{58} \mathrm{Co}$, and ${ }^{27} \mathrm{Al}(\mathrm{n}, \alpha)^{24} \mathrm{Na}$, using AJAX and PALEALE utility modules of AMPX working library. Reaction ${ }^{115} \mathrm{In}(\mathrm{n}$, $\left.n^{\prime}\right)^{115 m}$ In was derived from ENDF/B-VI.8, while reaction ${ }^{103} \mathrm{Rh}\left(\mathrm{n}, \mathrm{n}^{\prime}\right)^{103 m} \mathrm{Rh}$ was not derived because there is no single or combination of reaction type numbers [18] (MT numbers in $\mathrm{ENDF} / \mathrm{B}$ ) for computation of production rate of metastable isomer of rhodium (first excited state resulting from inelastic scattering). The obtained multigroup cross-sections without rhodium are Doppler and resonances corrected and have MAVRIC shielding structure of $200 \mathrm{n} 47 \mathrm{~g}$. These reactions were also defined as a response functions for FW-CADIS methodology.

Two different approaches for the MAVRIC source distribution were

(1) fission distribution in space and energy determined by KENO-VI calculation (transferred as a source input into MAVRIC)-CAAS case,

(2) Watt spectrum [5] for source energy distribution $p(E)=C e^{-E / a} \sinh (\sqrt{b E})$ (we used thermal fission of ${ }^{235} \mathrm{U}$ with $a=1.028 \mathrm{MeV}$ and $b=2.249 / \mathrm{MeV}$ ) with uniform space sampling restricted to the reactor core-Watt spectrum case.

The total number of neutron batches was 2000 with 2000 neutrons per batch for MAVRIC sequence. The Denovo $S_{N}$ mesh had approximately 20000 cells over PCA facility model, that is, $48 \times 23 \times 18$ cells in $x y z$ direction with average cell side of $3.2 \mathrm{~cm}$, while Monaco Monte Carlo mesh had 36000 cells $(60 \times 30 \times 20)$ with average cell side of $2.6 \mathrm{~cm}$. We used $S_{8}$ as quadrature order and $P_{3}$ for Legendre order of scattering cross-section expansion (upscattering was deactivated). Since the axial flux gradients inside tubes are confirmed to be sufficiently small, the user-selected height of $\pm 1.5 \mathrm{~cm}$ for volumetric cylindrical tally regions in MAVRIC calculation represents a good compromise between Monte Carlo relative error and spatial description of PCA benchmark facility. Differential small void cylinders were also placed in the midplane $(z=0)$ of the access tubes. Point detectors and region track length estimators were used to find multigroup

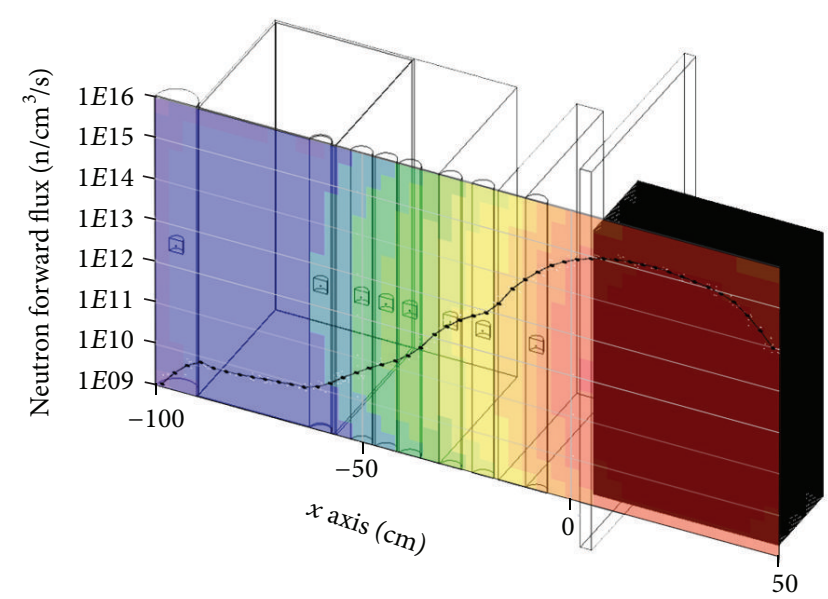

FIGURE 3: Denovo forward neutron flux axial distribution (front half removed).

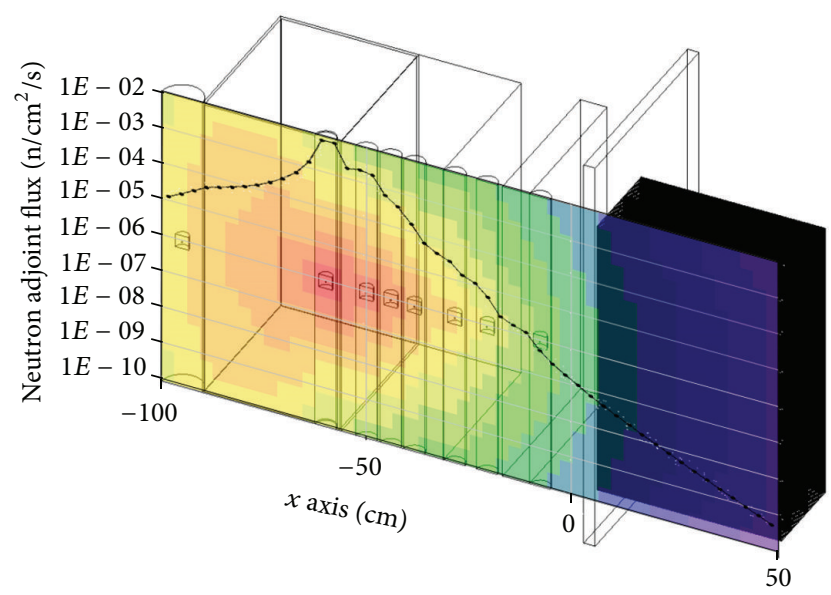

Figure 4: Denovo adjoint neutron flux axial distribution (front half removed).

neutron fluxes, reaction rates and finally equivalent fission fluxes.

The elements of the FW-CADIS methodology are depicted next. Forward and adjoint neutron flux axial distribution from Denovo code for CAAS case are shown in Figures 3 and 4, respectively. Biased source distribution and mesh-based importance map are shown in Figures 5 and 6, respectively. Total Monte Carlo neutron flux in form of mesh tally is depicted in Figures 7 and 8.

The calculated equivalent fission fluxes with Monte Carlo relative error (1 sigma level) for PCA Benchmark are given in Table 1 for MAVRIC CAAS case. Only the reactions for which the measurements were done are listed. Averaged $\mathrm{C} / \mathrm{M}$ ratios for detectors A1-A7 are shown in Figure 9 for CAAS case and in Figure 10 for Watt spectrum case. These results are compared to the referenced DORT results [2]. One can notice high similarity between Monaco results for two different source distribution approaches. The Watt spectrum case results are more conservative and they are $10-15 \%$ higher than CAAS case results. 


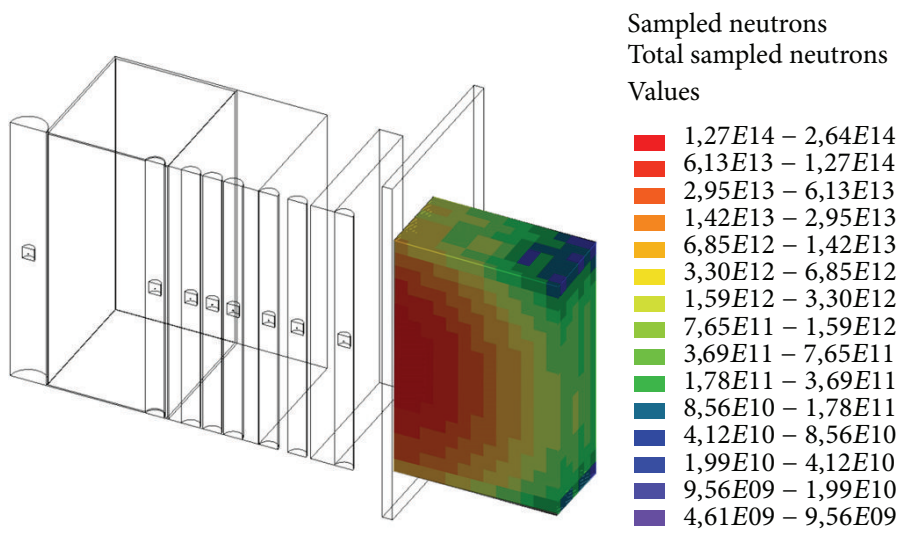

FIGURE 5: Biased source distribution (front half removed).

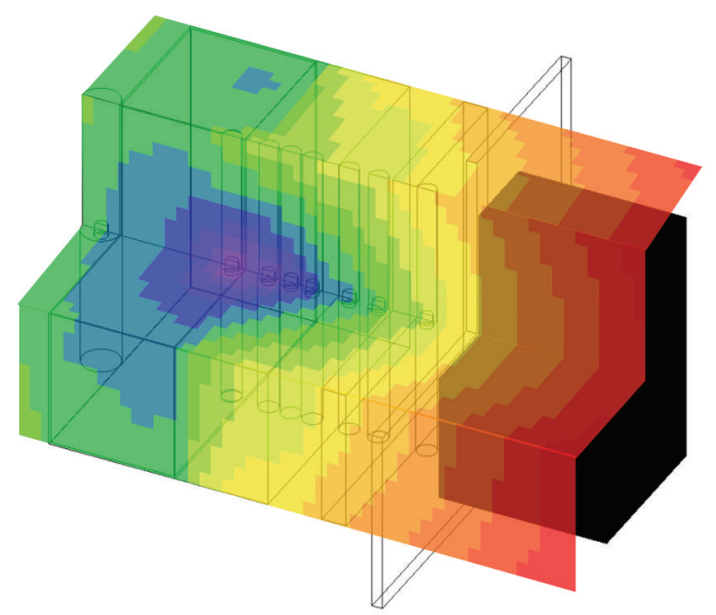

Neutron target

Group 1

Values

FiguRE 6: Mesh-based importance map (front quarter removed).

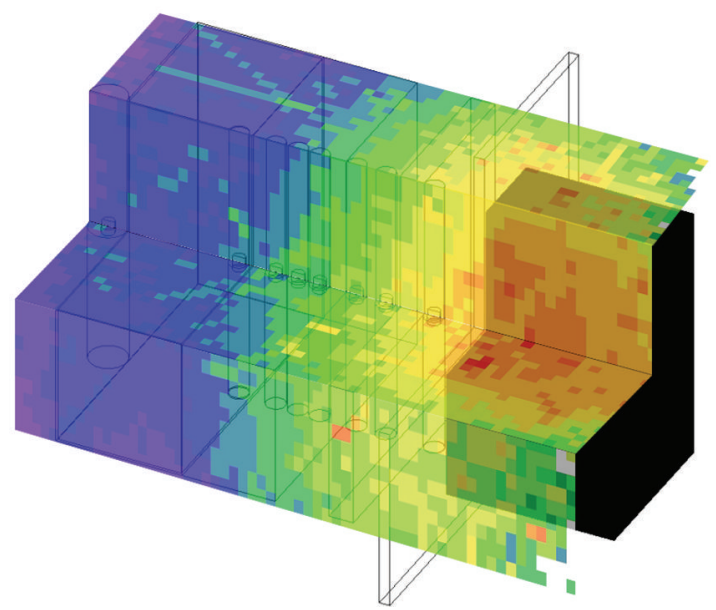

Neutron flux

Total neutron flux

Values

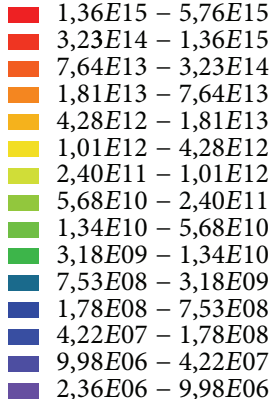

FigURE 7: Neutron flux mesh tally (front quarter removed).

\section{Discussion of Results}

All obtained results are in accordance with calculational uncertainty criterion from US NRC Regulatory Guide 1.190; the calculated reaction rates agree with the measurements to within $20 \%$ for out-of-core dosimetry locations. Under prediction in $\mathrm{C} / \mathrm{M}$ ratio can be observed for ${ }^{238} \mathrm{U}(\mathrm{n}, \mathrm{f}){ }^{137} \mathrm{Cs}$ (1.45 MeV threshold) through the thick RPV simulator (from locations A4 to A6), with 0.8 on average. High attenuation of neutron flux in that area (around 700 for IRDF-2002) will cause softening of neutron spectrum in RPV simulator, which shifts neutrons in resonance regions for inelastic scattering 


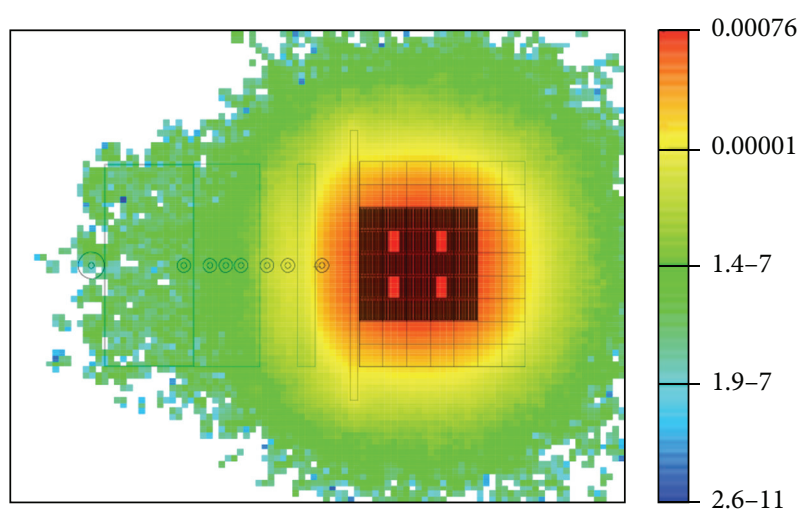

FIGURE 8: Neutron flux mesh tally in axial midplane (MCNP5 criticality results).

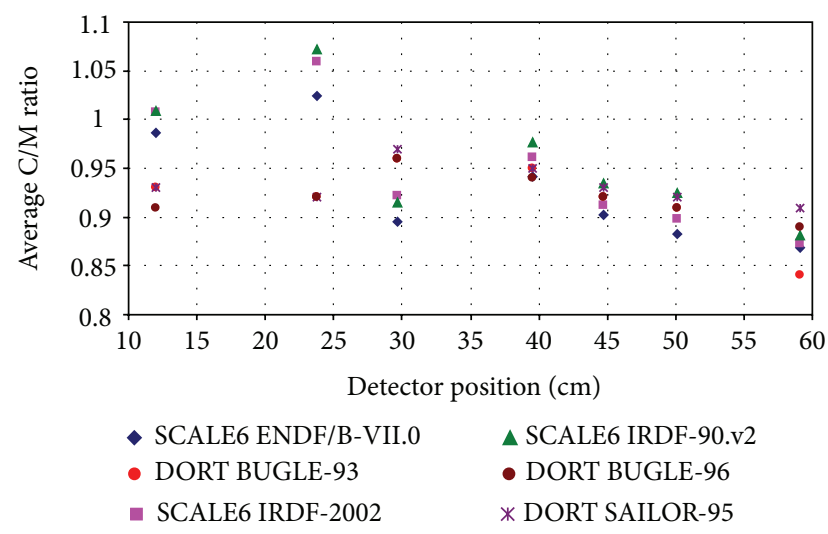

Figure 9: Average DORT [2] and SCALE6.0 (CAAS case) C/M ratios for detectors.

on iron. Microscopic cross-section for neutron inelastic scattering on iron is shown in Figure 11. Results for ${ }^{238} \mathrm{U}(\mathrm{n}$, f) ${ }^{137} \mathrm{Cs}$ indicate self-shielding effects, that is, sensitivity of multigroup shielding library v7 200n47g on the iron crosssections.

Overprediction in results is highest for detector A2, immediately after stainless steel thermal shield, which has large amount of iron. Again, self-shielding effects of iron cross-sections are pronounced, especially for ${ }^{27} \mathrm{Al}(\mathrm{n}, \alpha)^{24} \mathrm{Na}$ (IRDF-90.v2) with C/M of 1.17. The IRDF-90.v2 library generally gives the highest values, while SCALE6.0 v7 $200 \mathrm{n} 47 \mathrm{~g}$ library gives the lowest values of calculated fluxes. The IRDF2002 results are right in the middle, so this library would be favorable for RPV dosimetry calculations. The obtained calculational results show overall a good agreement with experimental results; however, for distant detectors from PCA core there is under prediction less than $10 \%$ on average, except excellent result for ${ }^{27} \mathrm{Al}(\mathrm{n}, \alpha)^{24} \mathrm{Na}$.

An important issue that has to be addressed when discussing Monte Carlo method is a burden the applied calculational model will place on memory resources and CPU time requirements. For that purpose the figure-ofmerit (FOM) factor [17] is defined to account for the time it

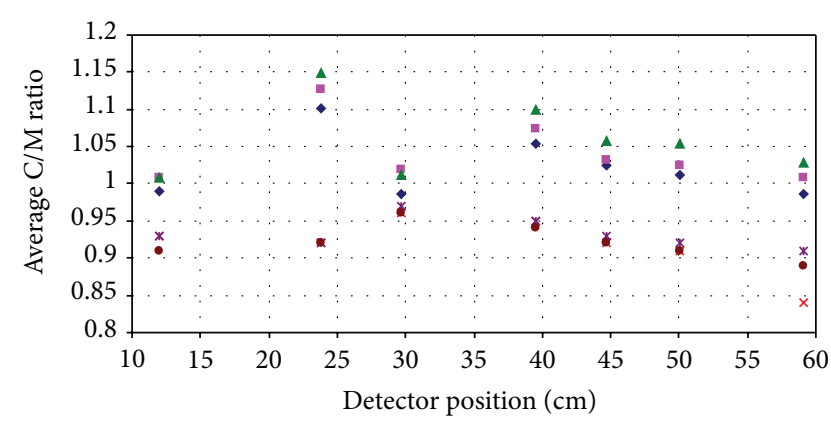

- SCALE6 ENDF/B-VII.0

* DORT SAILOR-95

$\times$ DORT BUGLE-93

- SCALE IRDF-2002

$\triangle$ SCALE IRDF-90.v2

- DORT BUGLE-96

Figure 10: Average DORT [2] and SCALE6.0 (Watt spectrum case) $\mathrm{C} / \mathrm{M}$ ratios for detectors.

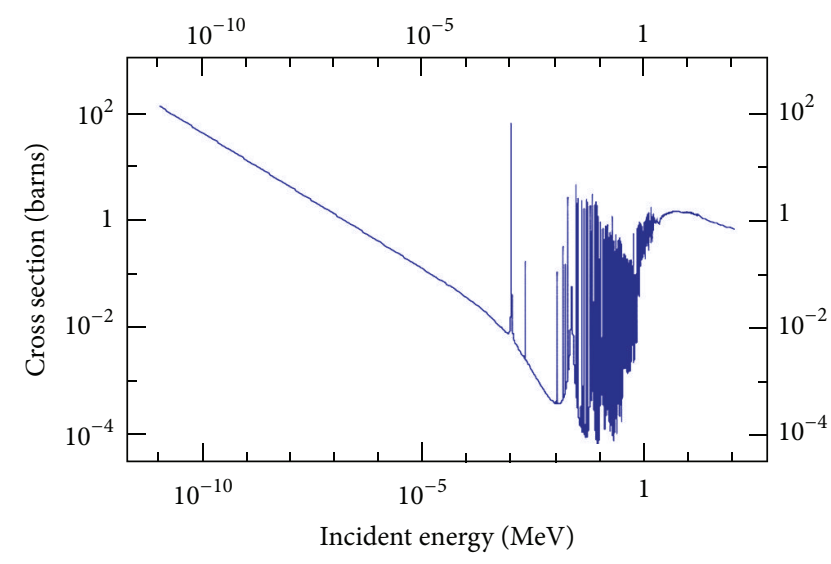

FIGURE 11: Inelastic scattering on iron [19].

takes to achieve a given level of uncertainty in Monte Carlo calculation,

$$
\mathrm{FOM}=\frac{1}{R E^{2} \cdot T},
$$

where $R E$ and $T$ are Monte Carlo relative error (on 1 sigma level) and Monte Carlo CPU run time (in min), respectively. MAVRIC run time for highest energy threshold reaction $\left(5 \mathrm{MeV}\right.$ for $\left.{ }^{27} \mathrm{Al}(\mathrm{n}, \alpha)^{24} \mathrm{Na}\right)$ was $7 \mathrm{~h}$, while for the lowest $\left(0.1 \mathrm{MeV}\right.$ for $\left.{ }^{103} \mathrm{Rh}\left(\mathrm{n}, \mathrm{n}^{\prime}\right)^{103 m} \mathrm{Rh}\right)$ was $10 \mathrm{~h}$. In this paper all calculations have been performed on QuadCore Q6600 with 8 GB of RAM. FOM factors for IRDF-2002 and CAAS case are shown in Figure 12. Application of automated variance reduction technique based on adjoint fluxes removes the burden of manually tuning VR parameters and significantly improves the quality of Monte Carlo calculations.

\section{Conclusion}

The analysis of the PCA benchmark has been performed using the SCALE6.0 code package. The calculational models of the PCA benchmark within CSAS6 and MAVRIC sequences of SCALE6.0 have been determined. The results 


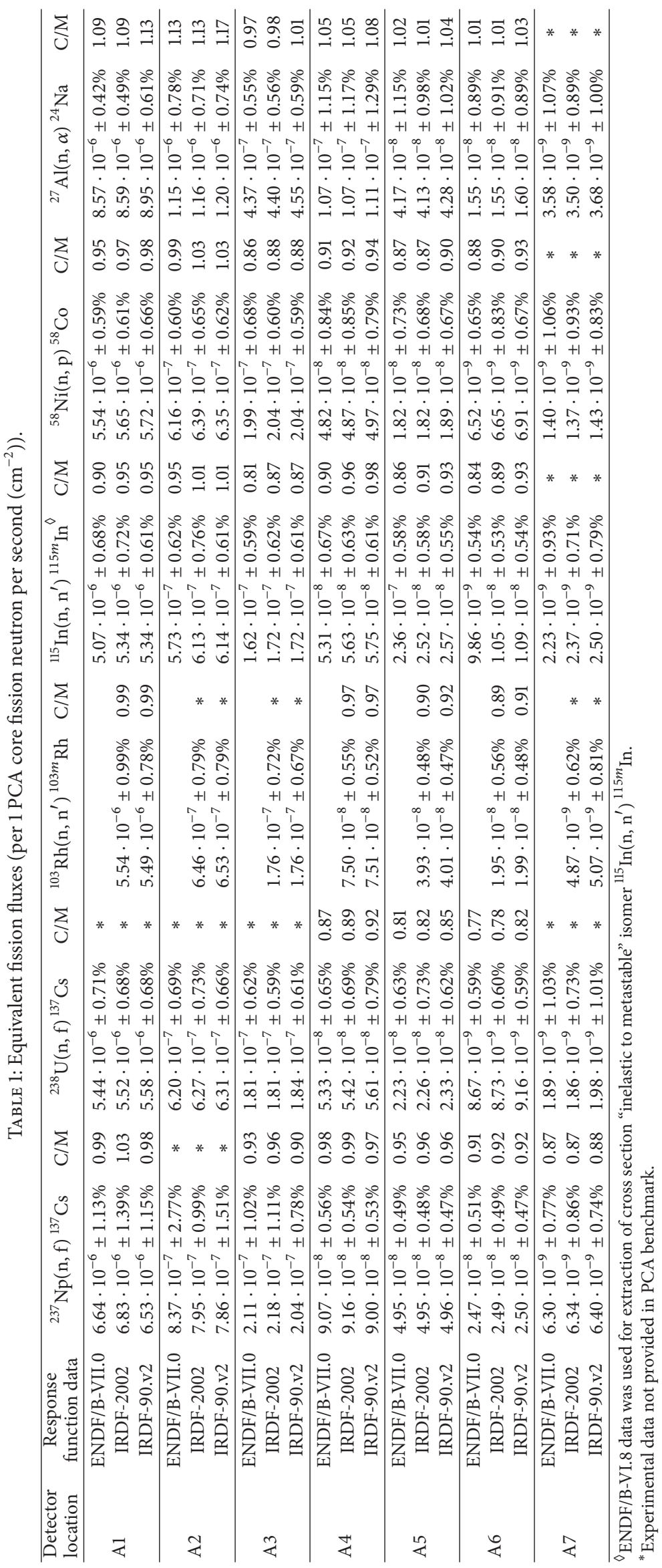




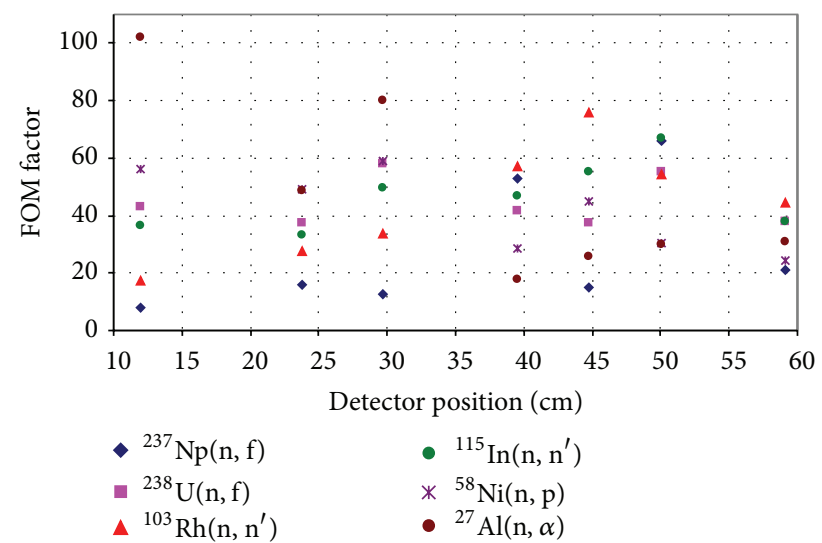

Figure 12: FOM factors for IRDF-2002 (CAAS case).

of calculations using the established models have been compared with PCA benchmark data. A good agreement of the calculated and measured equivalent fission fluxes has been obtained. No systematic decrease of agreements between calculations and measurements with increasing distance of detector from the PCA benchmark facility core was observed for any of the applied approaches. This indicates that the shapes of calculated neutron spectra, in the energy range were dosimeters are sensitive, are properly determined. SCALE6.0 capabilities in generation/mixing cross-sections from working library v7_200n47g, to be used as a response functions in MAVRIC, proved to be very useful.

\section{References}

[1] "Shielding Integral Benchmark Archive and Database," SINBAD Reactor Shielding Benchmark Experiments, OECD/NEA, 2011.

[2] I. Remec and F. B. Kam, "Pool Critical Assembly Pressure Vessel Facility Benchmark," NUREG/CR-6454 (ORNL/TM-13205), prepared for the U.S.NRC by Oak Ridge National Laboratory, August 1997.

[3] A. H. Fero, S. L. Anderson, and G. K. Roberts, "Analysis of the ORNL PCA benchmark using TORT and BUGLE-96," in Reactor Dosimetry: Radiation Metrology and Assessment, J. G. Williams, D. W. Vehar, F. H. Ruddy, and D. M. Gilliam, Eds., pp. 360-366, American Society for Testing and Materials, Philadelphia, Pa, USA, 2001.

[4] Y. K. Lee, "Analysis of the NRC PCA pressure vessel dosimetry benchmark using TRIPOLI-4.3 Monte Carlo code and ENDF/BVI.4, JEF2.2 and IRDF-90 libraries," Nuclear Mathematical and Computational Science: A Century in Review, A Century Anew, Gatlinburg, Tenn, USA, April 2003.

[5] "SCALE: A Modular Code System for Performing Standardized Computer Analyses for Licensing Evaluation," ORNL/TM2005/39 Version 6, Radiation Safety Information Computational Center at Oak Ridge National Laboratory, 2009.

[6] D. Vragolov, M. Matijević, and D. Pevec, "Modeling of pool critical assembly pressure vessel facility benchmark," in Proceedings of the International Conference Nuclear Energy for New Europe, Ljubljana, Slovenia, 2010.
[7] J. M. Risner, D. Wiarda, M. E. Dunn, T. M. Miller, D. E. Peplow, and B. W. Patton, "Production and Testing of the VITAMINB7 Fine-Group and BUGLE-B7 Broad-Group Coupled Neutron/Gamma Cross-Section Libraries Derived from ENDF/BVII.0 Nuclear Data," U.S.NRC NUREG/CR-7045, 2011.

[8] International Atomic Energy Agency, Vienna, Austria, International Reactor Dosimetry File 2002 (IRDF-2002), 2006.

[9] International Atomic Energy Agency, Vienna, Austria, International Reactor Dosimetry File (IRDF-90 Version 2), 1993.

[10] U.S.NRC, Calculational and Dosimetry Methods for Determining Pressure Vessel Neutron Fluence, Regulatory Guide 1.190, 2001.

[11] I. Remec and F. B. K. Kam, "H.B. Robinson-2 Pressure Vessel Benchmark," NUREG/CR-6453 (ORNL/TM-13204), prepared for the U.S.NRC by Oak Ridge National Laboratory, October 1997.

[12] J. C. Wagner and A. Haghighat, "Automated variance reduction of Monte Carlo shielding calculations using the discrete ordinates adjoint function," Nuclear Science and Engineering, vol. 128, no. 2, pp. 186-208, 1998.

[13] J. C. Wagner, E. D. Blakeman, and D. E. Peplow, "Forwardweighted CADIS method for global variance reduction," Transactions of the American Nuclear Society, vol. 97, pp. 630-633, 2007.

[14] G. I. Bell and S. Glasstone, Nuclear Reactor Theory, Van Nostrand Reinhold Company, Litton Educational Publishing, 1970.

[15] J. C. Wagner, Acceleration of Monte Carlo shielding calculations with an automated variance reduction technique and parallel processing [Ph.D. thesis], Pennsylvania State University, University Park, Pa, USA, 1997.

[16] M. B. Chadwick, P. Obložinský, M. Herman et al., "ENDF/BVII.0: next generation evaluated nuclear data library for nuclear science and technology," Nuclear Data Sheets, vol. 107, no. 12, pp. 2931-3060, 2006.

[17] X-5 Monte Carlo Team, MCNP-A General N-Particle Transport Code, Version 5, vol. 1 of Overview and Theory, LA-UR-031987, Los Alamos National Laboratory, 2003.

[18] A. Trkov, M. Herman, and D. A. Brown, "ENDF-6 Formats Manual," Data Formats and Procedures for the Evaluated Nuclear Data Files ENDF/B-VI and ENDF/B-VII, National Nuclear Data Center, Brookhaven National Laboratory, 2011.

[19] IAEA Nuclear Data Services, http://www-nds.iaea.org/. 


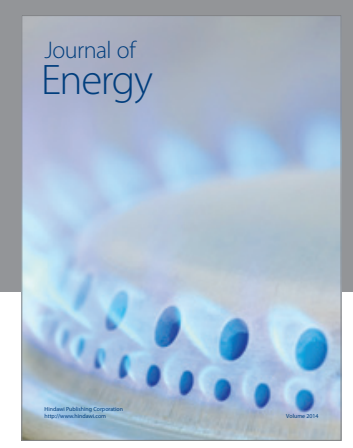

Journal of

Industrial Engineering
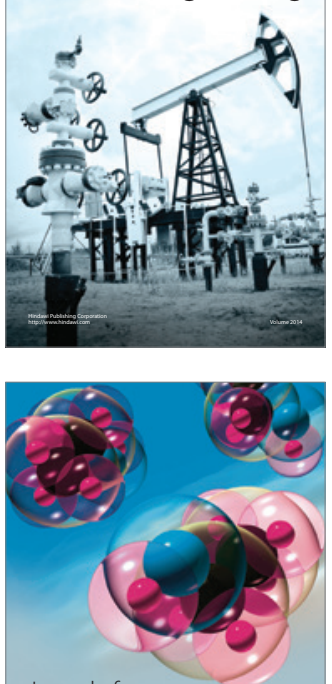

Fuels
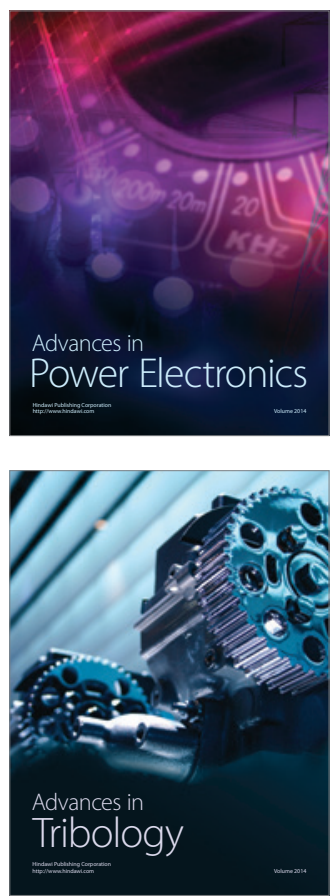

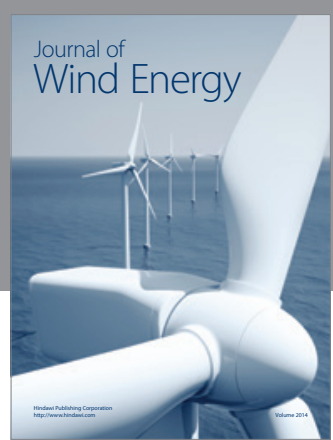

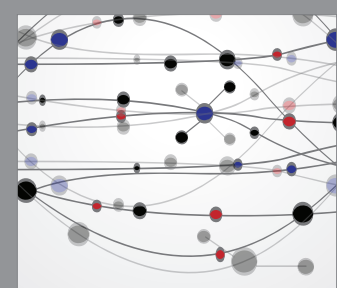

The Scientific World Journal

Submit your manuscripts at http://www.hindawi.com

Journal of

Structures
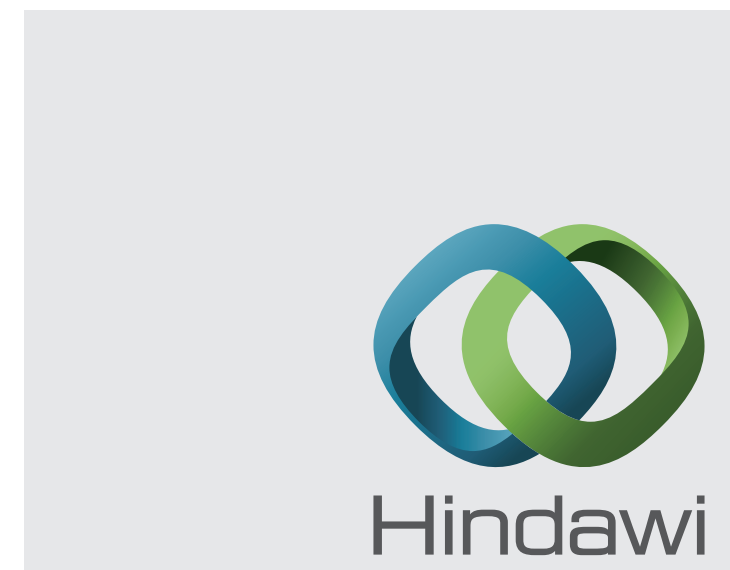

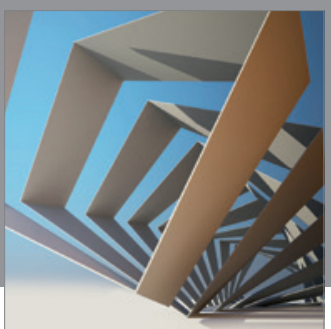

Rotating

Machinery
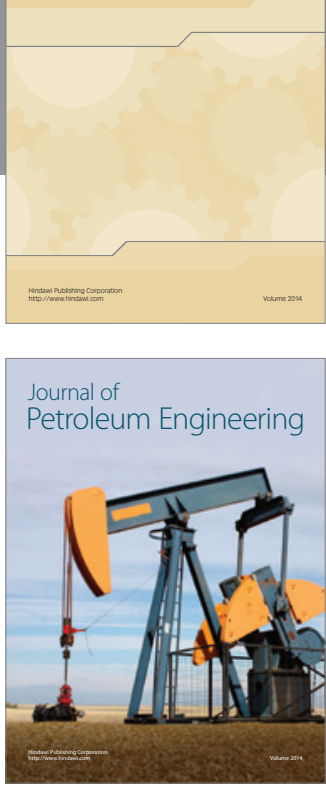

Journal of

Solar Energy
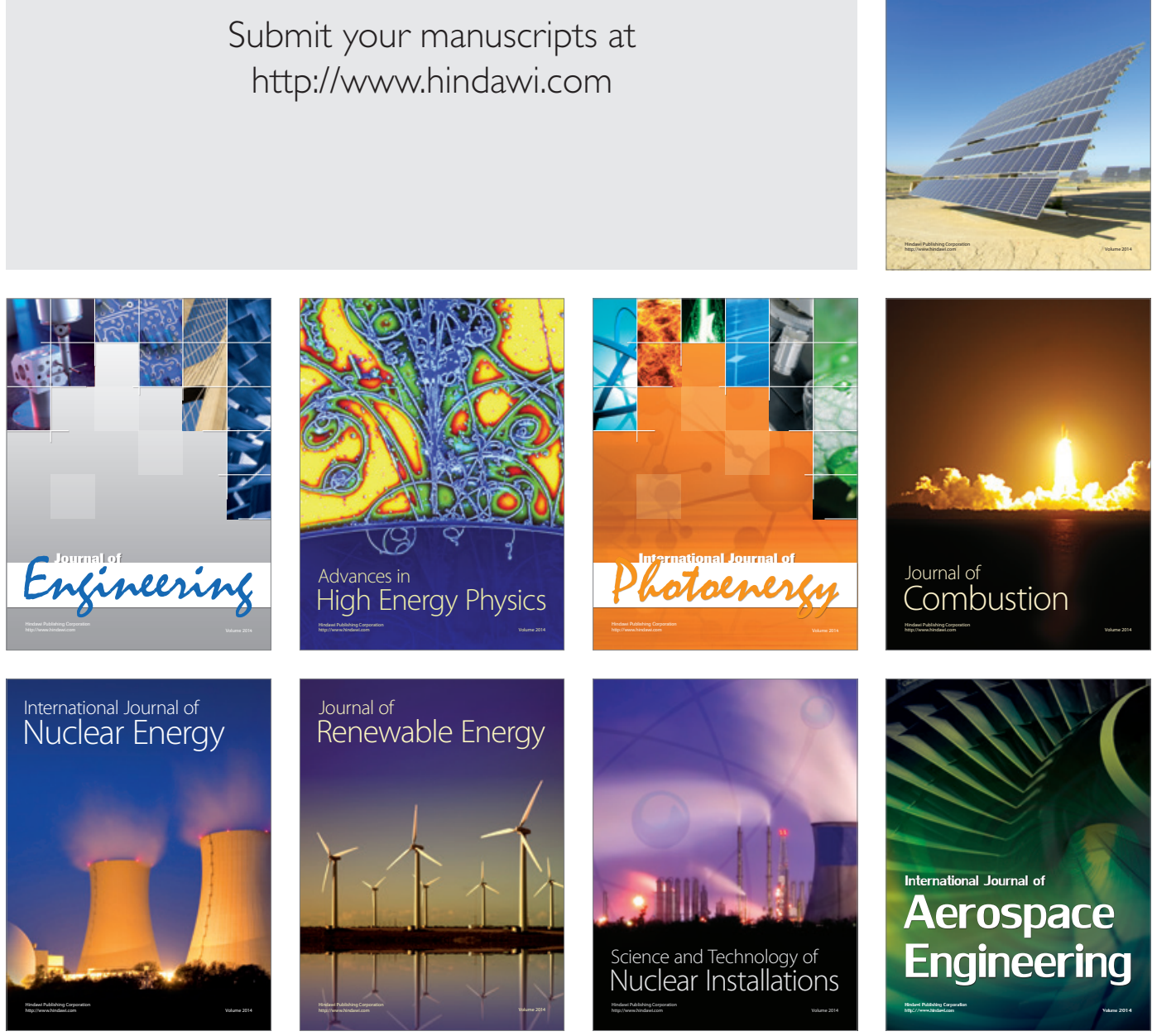